\title{
Téoros
}

Revue de recherche en tourisme

\section{Valorisation de l'environnement et tourisme. Le cas du Parc National des Pyrénées}

\section{Michel Bouvier, Marie Hervieu et Michel Balfet}

Volume 14, numéro 1, printemps 1995

Les parcs : des réseaux en mutation

URI : https://id.erudit.org/iderudit/1077061ar

DOI : https://doi.org/10.7202/1077061ar

Aller au sommaire du numéro

Éditeur(s)

Université du Québec à Montréal

ISSN

0712-8657 (imprimé)

1923-2705 (numérique)

Découvrir la revue

Citer cet article

Bouvier, M., Hervieu, M. \& Balfet, M. (1995). Valorisation de l'environnement et tourisme. Le cas du Parc National des Pyrénées. Téoros, 14(1), 60-66.

https://doi.org/10.7202/1077061ar 


\section{Valorisation de l'environnement et tourisme. Le cas du Parc National des Pyrénées}

Michel Bouvier, Marie Hervieu et Michel Balfet"

Dans l'actualité des politiques d'aménagement du territoire et de développement durable des zones rurales, il est logique de voir un lien entre tourisme et protection des espaces naturels. Autour de l'exemple particulier du Parc National des Pyrénées, il convient de rappeler que si la question se pose en termes d'avenir économique, elle est déjầ présente en termes de gestion. C'est en effet le Parc National le plus *fréquentés de France avec plus de 2 millions de visites par an, qu'il doit sans doute à son image de conservatoire naturel, mais qu'il tenait déjà avant sa création en 1967 de la notoriété de quelques sites tels le Cirque de Gavarnie et le Pont d'Espagne.

Quoi qu'il en soit, la valorisation de l'environnement par le tourisme ne saurait être affirmée d'emblée sans précautions, et il serait tentant d'inverser la relation en constatant un désir et une possibilité de valorisation du tourisme par l'environnement. Autrement dit, la question doit-elle être abordée d'abord sur le plan qualitatif ou privilégier la notion de chiffre d'affaires? Sur ce plan là, il est alors raisonnable d'associer une notion de valeur au patrimoine naturel protégé. Que cette valeur puisse prendre une dimension économique, c'est indéniable, mais l'on pourrait en outre, considérer qu'elle apporte à la politique de préservation une garantie supplémentaire de pérennité.

\section{Réalités du Parc National des Pyrénées}

\section{Objectifs de ses promoteurs}

Espace protégé pour tous et pour les générations à venir, il s'inscrit dans un réseau mondial des pares nationaux, témoin des ambitions que s'est donnée la France pour

Monsieur Michel Bouvier est docteur véterina ire inspecteur en chef et directeur du Parc National des Pyrénées, Madame Marie Hervieu est chargée de mission aDéveloppement tourismen au Parc National des Pyínbes. Monsieur Mictie Balfet est maître de conférences en sciences et gestion ef directeur du departement hótellerie, tourisme, thermalisme (CETLA) a l'Unversite Tou. louse-Le Mirail. protéger ses milieux naturels les plus précieux. Mais pour le département des Hautes Pyrénées il est aussi un atout au service d'une politique d'aménagement dụ territoire et de développement durable.A la fois conservatoire d'un patrimoine naturel de valeur, terre d'accueil d'un tourisme de nature qui se développe, et image d'une activité traditionnelle qui l'a façonné et qu'il invite à maintenir, le Parc, établissement public national à caractère adminis= tratif, remplit les missions qu'il tient de la lol du 22 juillet 1960 et de son décret de création du 23 mars 1967 à savoir:

- protéger et mieux connaître les richesses faunistiques, floristiques, paysagères, mais aussi culturelles qui le caractérisent;

- accueillir un large public et lui enseigner un comportement de respect vis-î-vis de la nature;

- contribuer au développement loçal et notamment à la mise en valeur des communes dites périphériques.

đJeune de 27 ans $\%$, modeste avec ses 450 $\mathrm{km}^{2}$, le Parc National des Pyrénées reste proche de l'homme: le berger qui a entretenu ses paysages en y amenant son troupeau, lecitadinquile découvreetle parcourt avec passion, le garde-moniteur qui pour mieux le gérer va à la rencontre des deux autres. Il en wa souvent ainsi des pares d'Europeoccidentale, mêmes'ils partagent l'objectif universel de conservation de la biodiversité, rappelée au sommet de Rioen 1992 , au mềme titreque ceux des continents les plus étendus et que le premier d'entre eux créé en 1872 aux États-Unis, le Parc National de Yellowstone, fort de ses 8800 $\mathrm{km}^{2}$.

Un parc national montagnard à la frontière de l'Espagne

Le Parc National se situe toujours audessus de 1000 mètres d'altitude jusqu'au point culminant des Pyrénées françaises, lä Pique longue du Vignemale à près de 3300 mètres. Il s'étend de la Neste d'Aure à la vallée d'Aspe, aux deux tiers sur le département des Haures-Pyrénées.
Sa diversité géologique de calcaire à Gavarnie, de granite à Cauterets, de volcanisme éruptif au Picdu Midid'Ossau, unie dans un passé glaciaire qui a créé 230 lacs, où l'influence croisée du climat atlantique et méditerranéen ont créé des paysages admirableset favorisélemaintiend'une flore variée comprenant quelques 150 espèces endémiques telle la $\approx$ Ramonde des Pyrénées* qui évoque la mémoire de Ramon, naturaliste passionné, comme ce massif a su en susciter.

L'homme aussi a créé des paysages, en défrichant la forêt pour le pâturage, et en laissant les traces d'un bấti pastoral traduisant la constance decette activitédans la région.

Une faune spécifique, qui a pu se maintenir jusqu'à nous, peuple ces habitats qu'une récente directive européenne nous invite davantage à protéger. Le desman, insectivore aquatique proche de la taupe, strictement pyrénéen et cantabrique, est sans doute l'exemple type du patrimoine précieux confié à la garde du Parc et des Pyrénéens.

L'ours est cependant plus en vue: sa conservation en Europe occidentale sera le résultat d'une attitude responsable. Quelques individus subsistent comme un espoir en Béarn, souvenir de leurs congénères disparus de Bigorre il $y$ a une vingtaine d'années.

Mais les espèces symboles du Parc National sont encore l'isard, dont l'interdiction de la chasse a permis de reconstituer une population de 4500 têtes, le grand tếtrasou coq de bruyère, les rapaces prestigieux que sont le gypaète barbu à l'envergure de 3 mètres, le vautour fauve ou l'aigle royal. Conservation et gestion active se complètent, notamment avec l'introduction de la marmotte qui ravit volontiers les randonneurs et le projet d'un retour du bouquetin ibérique, dont les derniers survivants dans les Pyrénées vivent à Ordesa.

La Brèche de Roland estl'imagedu lien qui unit le Parc National des Pyrénées et son 
voisin espagnol, le Parc National d'Ordesa etduMont Perdu. Une charte de coopération signée en 1988 prépare la voie à un Parc International. Mais c'est depuis de nombreux siècles que traités, lies et passeries, manifestent $l^{1}$ échange des populations et activités montagnardes entre Barèges et Broto, entre Aspe et Hecho ou, plus près de nous, que les passages des républicains espagnols et des volontaires de la France libre relativisent la notion de frontière-barrière.

\section{Un établissement public national d'implantation locale}

Parmi les dispositifs de protection de l'espace, celui des Parcs Nationaux est le plus complet, mettant en oeuvre une réglementation mais aussi une structure de gestion: un établissement public national à caractère administratif.

Cette structure sous la responsabilité d'un directeur s'appuie sur deux des rares corps de fonctionnaires du Ministère de l'Environnement: les agents techniques et les techniciens des parcs nationaux, gardesmoniteurs, qui interviennentgénéralement sur le terrain, et sur des services techniques d'appui au siège, lui même situé à Tarbes, le Préfet des Hautes-Pyrénées étant le commissaire du gouvernement auprès du Parc,

Unconseil d'administration de 50 membres vote le budget et oriente la politique du Parc. Ses délibérations sont soumises à l'approbation ministérielle. Il est actuellement présidé par un élu local et comprend 20 représentants des mairies, des conseils généraux et régionaux concernés, 9 représentants de services de l'État, et des personnalités qualifiées au titre de la protection de la nature, de la chasse, de l'agriculture, des métiers de la montagne, etc.

Une commission permanente de 10 membres agit par délégation et intervient notamment dans le traitement de l'indemnisation des dégâts d'ours aux troupeaux domestiques. Un comité scientifique de 18 membres apporte son concours, par des avis ou des propositions de recherche, à l'action du Parc. Il doit couvrir les spécialités intéressant le territoire protégé, y oompris les sciences humaines, mais aussi permettre le lien avec les milieux universitaires et de la recherche.

\section{Un acteur du développement local}

Au côté de son service scientifique et de ses missions de police de la nature, le Parc ouvre son action à divers domaines, vers différents partenaires.

Un service «développements, un service "pastorialismes, un service *communicationsoeuvrenten faveur et, avecles bergers, les offices de tourisme, les municipalités de la zone périphérique.

Le Parc National des Pyrénées participe, par exemple, à l'animation de la Maison de la Vallée à Luz, au sein d'un syndicat mixte, à l'aménagement du site du Pont d'Espagne, associéà la Société d'Economie mixte, à la modernisation des conditions du travail pastoral en concourantà l'amélioration des locaux de fabrication du fromage, de logement des bergers et, en organisant l'héliportage annuel du matériel nécessaire à la sison d'estive.

Le contrat de plan État-Région est le support d'une mise en valeur de la zone périphérique, mettantà disposition du Parc des crédits permettant d'aménager, d'amé iorer l'accueil, d'embellir villages et paysages, et de faire vivre un pays fidèle à l'image de nature protégée.

Parmi les priorités du programme quinquennal d'aménagement du Parc National, on trouvera notamment l'importance de gérer une surfréquentation ponctuelle des grands sites comme Gavarnieou Cauterets, et d'en éviter ainsi une dégradation dommageable. Les visiteurs du parc sont d'ailleursdansleurgrandemajoritépréparés à un recul sensible de la civilisation automobile au profit d'un accueil respectueux de la richesse du patrimoine paysager et naturel qui leur est offert.

L'ambition du Parc est sans doute d'associer à sa démarche, avec ses partenaires, une zone périphérique mise en valeur à partir desonidentitémanifeste, la montagne de Bigorre et Bearn, et de ses richesses patrimoniales. Le Parc est un trait d'union naturel, dans le sens de la politique de massif. Il relie haute et basse vallée, les vallées entre elles, habitants montagnards et visiteurs citadins, et même deux départements et deux régions administratives.

Son action pédagogiqueva nécessairement vers les générations futures, depuis l'école des vallées jusqu'au centre d'accueil des classes déplacées. Il la développe dans des livvres, des films et des conférences, ainsi qu'à travers des expositions locales, base d'une éducation à la Nature et d'une sensibilisation au respect de ce patrimoine.

\section{La fréquentation touristique du Parc National des Pyrénées}

Dès la fin du $18^{\circ}$ siècle, les Pyrénées connaissent un véritable engouement et il est de bon ton de fréquenter ses stations. Dans le sillage de l'ascensionnisme mondain commence l'époque du Pyrénéisme. Gavarnie tout naturellement, comme Chamonix ou Zermatt aura ses guides, ses valeureux précurseurs.

La clientèle aristocratiqueenvoûtée par les bienfaits des eaux thermales et du climatisme viendra volontiers se faire voir dans les stations à̀ la modes. Les romantiquesdu $19{ }^{\circ}$ siècle feront des Pyrénées leur montagne de prédilection et maintes proses fleuriront de ces sites grandioses.

Le monde du $19^{\circ}$ siècle sera relayé par la fréquentation d'une nouvelle clientèle. Les vacanciers aux congés payés etles nouveaux curistes se mettent à leur tour à fréquenter les stations pyrénéennes. La fréquentation de la montagne pyrénéenne devient plus nombreuse, plus banale. Cette fréquentation concerne des sites et des paysages qui seront dès 1967 partie intégrante du Parc National des Pyrénées et, par là même, espaces protégés.

Dès lors se pose le problème de la connaissance des flux touristiques pour une meilleure gestion et protection de ces espaces protégés.

Le Haut Comité pour l'Environnement lançait en 1975 un appel d'offres auquel répondaientdeschercheursdel'Université de Bordeaux pour mesurer et qualifier ces flux... Le Parc National des Pyrénées était lepremier supportd'une telleinvestigation. Le constat dresséà l'époqueétaitlesuivant:

On connaissait mal l'utilisation qui était faite du Parc en tant qu'équipement, et, d'autre part on s'interrogeait quant à la possibilité de gérer simultanément deux missions propres aux Pares Nationaux: la protection de la nature et l'accueil du public, notamment sur des sites déjà bien fréquentés. Cette étude permettait alors une meilleure connaissance de la fréquen- 
tation et la mise en place de nouvelles orientations d'aménagement.

17 ans après, il est apparu opportun et nécessaire de réaliser une nouvelle enquête. Les résultats de cette étude permettraient d'orienter les politiques à venir en matière de gestion et d'ouverture de cet espace naturel protégé. Les préoccupations du gestionnaire PNP étaient les suivantes:

- mieux orienter les flux de fréquentation;

- mieux répondre aux attentes des visiteurs actuels et potentiels;

- sensibiliser les socio-professionnelset les institutionnels à l'importance du phénomène touristique dans et induit par le Parc National des Pyrénées;

- proposer de nouvelles perspectives en matière de développement économique et touristique;

- mettre en place un observatoire minimum qui permette de mesurer les principales évolutions de façon permanente.

L'enquête a été réalisée sur l'ensemble de la zone centrale ainsi que sur la Réserve naturelle du Néouvielle gérée par le Parc National des Pyrénées. L'étude de fréquentation de 1992 a comporté une étude quantitative et une étude qualitative sur deux saisons touristiques, été 1992 et hiver 1992-93(1). L'étude quantitative a permis d'appréhender les volumes globaux de fréquentation, d'évaluer la perte de charge de l'accès routier jusqu'au sentier et d'analyser les flux dans le temps et dans l'espace. Des comptages ont été effectués sur les parkings et sur les sentiers et des compteurs routiersont été mis en placesur l'ensemble des accès (26routes). Le nombre de véhicules stationnés à 14 h sur les parkings a été relevé ainsi que le numéro du département d'origine ou le pays étranger. Cescomptagesontété effectués sur 4 aprèsmidi de juillet et d'août. Les comptages sur les sentiers ont eu lieu les mêmes jours que les comptages sur les parkings. Les montées et les descentes heure par heure, de 9 $\mathrm{h}$ à $19 \mathrm{~h}$, ont été comptabilisées. Les oomptages routiers ont été réalisés du 15 juin au 15 septembre, soit en mode horaire, soit en mode journalier.

L'étude qualitative avait pour objectifs:

- la connaissance des visiteurs et des usagers;
- l'identification de leurs comportements par rapportà l'espace, auxaménagements et aux équipements;

- la connaissance de leurs motivations et leurs attentes tant en matière d'aménagement qu'en matière d'animation;

- une première approche des attentes de la clientèle de la saison hivernale et de la basse saison.

Ces différentes données ont été recueillies à l'aide de questionnaires proposés à des randonneurs (enquête sur les sentiers) et des epique-niqueurs" (enquête sur les parkings). 400 questionnaires ont été remplis sur les parkings et 1700 personnes ont été interrogées sur les sentiers. En fonction des sites (sites à forte fréquentation, sites à faible fréquentation), le poids d'enquête était différent.

\section{L'analyse de la fréquentation} sur le Parc National des Pyrénées

Du 15 juin au 15 septembre 1992, la fréquentation du Parc National a été estimée à 1772000 visites à partir de 575000 véhicules qui ont pénétré ou approché le territoire du Parc, cela à raison de 3,08 personnesparvéhicule. Cette fréquentation est la plus importante des Pares Nationaux de France, indépendamment des différences de surface, d'accès, d'intérêt touristique et d'économie locale.

Cependant, il faut noter une très grande variabilité des flux selon les sites. Cette variation est due essentiellement à la notoriété des sites. Ainsi, Gavarnie et Gaube représententà eux seulsla moitiédu nombre des randonneurs. Le flux affectant un site peut également être rapproché du nombre des hébergements banalisés dans la vallée. On note peu de passages d'une vallée à l'autre, la moitié des personnes séjournent dans la vallée du Parc où elles randonnent.

L'ensembledes visiteurscomptabilisépeut être classé sous deux types: les randonneurs et les contemplatifs, Ces derniers sont trois fois plus nombreux que les randonneurs. Seulement $35 \%$ ont emprunté les sentiers à plus de 15 minutes d'un parking.

L'intensité des flux doit également être rapprochée d'autres conditions telles: la météo (le Parc est fréquenté 11 fois moins un jour de pluie qu'un jour de soleil), la période ( $48 \%$ des visites ont été réalisées pendant le mois d'août - $38 \%$ en juillet), les jours (week-end - semaine).

\section{Qui sont les visiteurs fréquentant le} Parc National des Pyrénées?

Les visiteurs du Parc National de Pyrénées sont originaires de l'Ouest de la France et surtout des régions les plus proches: les régions d'Aquitaine et Midi-Pyrénées, ces dernières représententla moitiédes visiteurs en été et les $2 / 3$ en hiver. Les étrangers des pays du Nord sont les plus nombreux en été, les espagnols en hiver.

Le public du Parc National des Pyrénées présente un caractère fortement régional en raison de la concurrence des Alpes. La grande majorité des visiteurs du Parc sont des touristes en vacances. Ils représentent une clientèle fidélisée. Les néophytes en première visite ne sont qu'un tiers en été et 1/4 en hiver. Ainsi, l'augmentation de la fréquentation du Parc est le fait à la fois de fidélisation des néophytes et de l'augmentation du nombre de visites dans le parc par séjour.

La principale raison de la venue des touristes sur le secteur du Parc National des Pyrénées est la découverte des Pyrénées (36\% des personnes). On ne peut pas parler véritablement d'un «public Paro car l'importance des habitués tient donc avant tout à la situation géographique et à l'habitude de venir en vacances dans la région. Cependant, il est certain que l'existence du Parc constitue un attrait supplémentaire aux yeux des touristes.

Les visiteursdu parcNationaldes Pyrénées ont une moyenne d'âge de 40 ans. On trouve davantage d'hommes $(69 \%)$ que de femmes. Ils appartiennent à des catégories socioprofessionnelles actives et aisées et ont un niveau d'études élevé.

\section{Les pratiques de montagne}

L'étalement de la durée de randonnée traduit la diversité des pratiques (de la marche promenade à l'alpinisme).

$32 \%$ des randonneurs marchenten famille et $40 \%$ randonnent en couple.

Le portrait type du randonneur est le suivant:

- il vient pour contempler le paysage et se détendre $(89 \%)$; 
- il a un appareil photo $(73 \%)$, des cartes ou guides de randonnées (47 $\%)$;

- il porte des chaussures de montagne $(53 \%)$ ou de sport (42\%).

Les éléments les plus appréciés dans le Parc National des Pyrénées sont avant tout des éléments liés à la géographie des lieux, bien avant la faune et la flore. Ce que le public apprécie avant tout, c'est ce qu'il a pu voir.

L'espace Parc National des Pyrénées est bien perçu par le public qui le reconnaît comme un organisme public national. $\mathrm{La}$ quasi-totalité des visiteurs sait qu'il existe une réglementation spécifique et l'accepte très bien. Le public se révèle très satisfait des aménagements actuels dans le Parc (parkings, sentiers). Les visiteurs ne sont guère favorables à de nouveaux aménagements, qui devront être, s'ils étaient réalisés, très respectueux de l'environnement. Les demandes en matière d'information sont constantes. Ils'agitessentiellement de demandes sur la faune et la flore et des panneaux explicatifs sur le paysage. Une demande plus spécifique et plus originale concernerait un journal. Les maisons du Parc sont peu connues et très peu utilisées. Grâce à cette nouvelle étude, une analyse de l'évolution sur les 15 dernières années de la fréquentation du Parc National des Pyrénées, des visiteurs et deson imagepeut être faite aujourd'hui. La fréquentation du Parc National des Pyrénées a augmenté globalement de $20 \%$ depuis 1975 avec des différences sensibles selon les sites. La population des visiteurss'est démocratisée etrégionalisée. Dans le même temps, cette population a quelque peu vieilli.

En moyenne, les visiteurs passent moins de temps dans le Parc mais les pratiques de fréquentation se sont diversifiées. L'accès à la nature par de nombreux usagers dans le respect des équilibres naturels tel qu'il s'est réalisé depuis 15 ans et le renforcement de son ancrage dans la région sont une réussite pour le Parc. L'image du Parc ne s'est pas altérée avec le temps.

D'autre part, le Parc National des Pyrénées joue un rôle essentiel auprès des collectivités locales. Sa présence contribue à attirer une clientèle au pouvoir d'achat élevé. Il appartient auxresponsables locaux (professionnels et institutionnels) de tirer profit à la fois de cette image positive liée aux Parcs Nationaux et de la présence de cet important gisement de clientèle.
Pour les visiteurs, la mission du Parc est claire: développer l'accueil des touristés en empêchant les aménagements contraires à l'idée de préservation de la nature.

Suite à cette analyse, nous pourrions résumer en quelques points le rôle que le Parc se doit de jouer désormais:

- mieux aocueillir le public en protégeant l'environnement;

- éviter tout nouvel aménagement contraire à cette protection;

- privilégier l'information.

Face à la saturation de certains sites soulignée dans l'étude, une réorganisation a été́ définie et est en cours de réalisation.

\section{Valorisation du patrimoine environnemental du Parc National des Pyrénées par un nouvel équilibre de la fréquentation touristique}

Une telle approche peutêtre perçuecomme provocatrice compte tenu des tensions ponctuelles apparues ici et là dans le massif pyrénéen ${ }^{(D)}$. Ces tensions alimentées par des considérations souvent généreuses, mais parfois éloignées des réalités quotidiennes, irritent bon nombre de ceux qui désirent vivre et travailler dans ces régions. A y regarder de plus près, et avec une volonté de dépassionner un débat riche en stéréotypes décalés, il peut émerger de l'analyse quel'opposition, fréquentation touristique - sauvegarde et maintien du patrimoine environnemental pyrénéen - n'est pas aussi nette que certains veulent l'affirmer.

Ce n'est pas cultiver le paradoxe que de partir d'une hypothèse différente, voire opposée, d'autant que le paradoxe tombe dès qu'on considère l'ensemble Pyrénéen et le Parc National des Pyrénées comme une globalité, un système ouvert et non comme une juxtaposition de systèmes clos dont individualités, associations ou groupes divers se seraient arrogés l'exclusivité d'un avisautorisésur leur étatet leur devenir.

Un système environnemental, et le terme «système» est là pour nous le rappeler, est un tout, un tout caractérisé par une infinité d'interrelations, mais un tout ouvert vers l'extérieur. Le Parc National des Pyrénées, la zone centrale, la zone périphérique sont liés à un piémont, une plaine, une région et deux entités nationales. Nier cela, c'est condamner l'idée même de Parç le con- ceptde réserve, nécessaire dans un cadre de considérations scientifiques, ne peut rester que l'exception exclusive.

Dans une perspective de *temps historiquem, les interrelations évoquées précédemment n'ont été que peu modifiées par les flux touristiques; par contre, recherche et développement industriel, exploitation intensive des ressources naturelles (eaubois-minerais) ${ }^{(0)}$, passage d'une ćconomie de subsistance rurale à une économie de marché, en fait, l'intégration del'ensemble pyrénéen aux systèmes régionaux et nationaux, ont profondément modifié le système environnemental et, par voie de conséquence, tous les sous-systèmes écologiques. Ce n'est pas le touriste qui a fait disparaitre l'ours, le seuil de disparition du plantigrade était atteint bien avant le développement de la fréquentation du massif par les touristes ${ }^{(4)}$.

Notre propos s'efforcera de présenter deux axes, matières à études, qui visent à nuancer des jugements, souvent péremptoires, portés sur une dualité jugée plutốt contre nature ${ }^{(9)}$ : tourisme et environnement:

- une synthèse des caractéristiques sociales et comportementales des visiteurs du Parc issue de l'étude de 1992 sur la fréquentation du Parc ${ }^{(6)}$. Certe approche permettra de formuler quelques remarques sur le niveau d'acceptation des contraintes de visites liées à la sauvegarde de l'environnement écologique;

- un essai d'identification des principaux axes de valorisation portés par la pratique du tourisme dans le Parc, particulièrement au niveau de la zone périphérique et du piémont.

Vers une acceptation et une appropriation des actions de régulation de fréquentation par les usagers du Pare

L'ensemble constitué par la zone centrale et la zone périphérique est d'une grande fragilité. Les risques induits par cette fragilité entraînent la mise en place de contraintes de comportement et d'accès pour les visiteurs. Ces contraintes ne peuvent être véritablement prises en compte par les usagers que si un phénomène d'appropriation psychologique intervient.

Letravail pédagogique réalisédans le cadre des structures du Parc a permis unepercep- 
tion et une compréhension des risques de surfréquentation de la part du plus grand nombre. Deux éléments ont facilité le processus:

- les caractères sociologiques, les attitudes et les comportements des usagers du Parc;

- l'avis des visiteurs du Parc des Pyrénées sur la régulation de la fréquentation

\section{Caractéristiques sociologiques et compor- tementales de visiteurs du Pare}

Deux familles de résultats statistiques ont été mis en évidence par l'étude de 1992 portant sur la fréquentation du Pare. Elles peuvent éclairer utilement les conditions et les circonstances qui ont permis et confortéle phénomène d'acceptation, puis d'appropriation des contraintes de régulation par les usagers.

Les caractéristiques sociologiques des visiteurs et les opinions qu'ils ont pu exprimer peuvent expliquer l'évolution rapide desmentalités dans le sens d'une régulation de fréquentation acceptée et intégrée par tous.

Origine géographique des visiteurs du Parc. Leurs modalités de sếour

Pour l'année 1992, l'origine géographique des visiteurs du Parc a été la suivante ${ }^{(\pi)}$, exprimée en pourcentage du total des visiteurs, (tableau $n^{\circ} 1$ ).

L'étude de l'origine géographique des visiteurs souligne deux aspects intéressants:

- l'importance du pôle d'émission constituépar les régionsà fort tropisme pyrénéen (régions de l'ouest et du sud-ouest);

- l'importance du tourisme de proximité au départ des zones proches du Parc (Midi-Pyrénées, Hautes Pyrénées, Pyrénćes Atlantiques).

Ces deux groupes représentent près de deux tiers de l'effectif des visiteurs; cette importance peut expliquer, tout au moins partiellement, l'appropriation rapide des mesures de régulation adoptées par les gestionnaires du Parc. Une bonne connaissance du milieu et des caractéristiques du Parc, de fréquents séjours, une proximité facilitante, sont autant de facteurs qui

\section{TABLEAU No 1}

Origine géographique des visiteurs du Pare

Etranger

Espagne

Autres pays

Ile de France

Rhônes Alpes

Zones de tropisme Pyrénéen

Bretagne

Loire

Poitou-Charentes

Aquitaine (sauf Gironde

et Pyrénées Atlantiques)

Guande

Languedoc-Roussillon

Zones liées à l'environnement du Parc

Midi-Pyrénées (sauf Hautes

Pyrénées et Pyrénées Atlantiques)

Hautes Pyrénées

Pyrénées Attlantiques
$3,37 \%$

$8,11 \%$

$7,93 \%$

$2,65 \%$

$33,59 \%$

3,82

8,00

5,71

5,73

8,13

2,20

$31,24 \%$

9,47

9,40

12,37
$64,83 \%-65 \%$ soit près de $2 / 3$ des effectif ont pu aider à l'intégration rapide de ces mesures dans l'univers cognitif, affectif et comportemental des usagerset des visiteurs.

Ces remarques peuvent être confortées par l'étude des caractéristiques sociales des visiteurs ${ }^{(b)}$. Le niveau d'études est largement plus élevé que la moyenne: plus de $35 \%$ de visiteurs ont fait des études supérieures, plus de $60 \%$ ont un niveau égal ou supérieur au baccalauréat. Professions intermédiaires, cadres moyens et supérieurs, professions libérales et étudiants représentent $56 \%$ de l'ensemble des visiteurs.

Les thèses sur la nécessité de protection de la nature et le maintien de l'équilibre environnemental trouvent dans la culture de tels visiteurs de larges potentialités de perception, de compréhension mais également de partage et de diffusion.

Les modalités de sĕjour dans l'environnement du Parc pezvent égalenent expliguer l'mplication des visitewrs

Les chiffres proposés sont issus d'une enquête portant sur la saison d'été 1992. Bien que très partiel, le caractère de ces résultats peut éclairer, malgré les risques de «réductionnismes, le niveau de réceptivité des visiteurs.

Plusieurs remarques peuvent être suggérées par les chiffres issus de cette étude.

L'importance du pourcentage des séjours moyens et longs ( $>3$ jours) atteint deux tiers de l'ensemble. Ils se déroulent essentiellement dans l'univers environnemental du Parc: zone centrale, zone périphérique ou Piémont. Ainsi présentent-ils deuxcaractéristiques économiques et sociales intéressantes: une implication directe du visiteur dans la vie et l'économie locale, associée à une durée significative de l'échange social.

L'implication économique du visiteur est un facteur non négligeable de maintien, voire de développement d'une activité permanente liée aux services d'accueil, de loisir, de commerce et d'artisanat. L'échange avec le tissu social local, amoroé le plus souvent par une simple demande d'information ou une transaction marchande va être enrichi par la durée. Son déroulement dans le temps et l'espace facilite la perception par le visiteur des réalités du milieu d'accueil, sa fragilité et la nature des limites qu'il faut accepter. La pédagogie par kimmersion* trouve ici une application quelque peu inattendue mais somme toute très opérationnelle.

\section{L'anis des visitewrs du Parc des Byrínées sur la régulation de la fréquentation}

L'enquête de 1992 déjà citée a permis de mettre en lumière l'avis des visiteurs sur le niveau de fréquentation du Parc et les modalités éventuelles de régulation.

- $70 \%$ de l'effectif interrogé et fréquentant le Parc trouve qu'il y a «assez de mondes ( $+6 \%$ par rapport à l'enquête de 1973); 
TABLFAU No2

Durée de séjour des visites au Parc

En \% du total des séjours

$\begin{array}{lr}\text { Résident } & 22 \% \\ \text { Durée }<3 \text { jours } & 12 \% \\ \text { Durée comprise entre } 3 \text { et } 7 \text { jours } & 23 \% \\ \text { Durée comprise entre } 8 \text { et } 15 \text { jours } & 25 \% \\ \text { Durée comprise entre } 16 \text { et } 31 \text { jours } & 15 \% \\ \text { Durée }>31 \text { jours } & 3 \%\end{array}$

TABLEAU N $\mathrm{N}^{\circ} 3$

Mode d'hébergement des visiteurs du Parc

en $\%$ du total des visiteurs

$\begin{array}{lr}\text { Camping bivouac } & 35 \% \\ \text { Refuge } & 8 \% \\ \text { Logement prêté ou loué } & 23 \% \\ \text { Hôtel } & 16 \% \\ \text { Résidence secondaire } & 4 \% \\ \text { Résidence principale } & 14 \%\end{array}$

- $27 \%$ estiment qu'il y a «trop de mondes (-2 \% par rapport à l'enquête de 1975).

$97 \%$ de l'effectif ainsi interrogé estime donc quela saturation est atteinte ou proche de l'être. Il convient de noter que seulement $1 \%$ de l'effectif interrogé est sans opinion.

Lorsque l'enquête aborde les moyens de régulation possibles, trois propositionssont suggérées:

- guide obligatoire:

$25 \%$ tout à fait d'accord ou assez d'acoord

$55 \%$ pas du tout d'accord ou pas d'accord

$10 \%$ sans opinion ou indifférent

- droit d'entrée:

$15 \%$ tout à fait d'accord ou assez d'acoord

$68 \%$ pas du tout d'accord ou pas d'accord

$27 \%$ sins opinion ou indifférent

- éloignement des zones de parcage:

$60 \%$ tout à fait d'acoord ou assez d'acoord

$10 \%$ pas du tout d'accord ou pas d'accord

$30 \%$ sans opinion ou indifférent

L'opposition majoritaire au guide obligatoire, l'opposition très marquée au droit Pyrénées
- maintien, voire développement d'une activité économique touristique qui s'ajoute aux activités traditionnelles dela zonepériphériqueet duPiémont;

- développement d'une image forte de qualité de vie, de conservatoire des traditions et de la nature.

Ces deux composantes constituent des aspects éminemment positifs de cette fréquentation touristique régulée et contrôlée. Déséconomies internes et externes, contre images sont progressivement réduites, laissant la place à une vision positive du Parc, aussi bien pour les visiteurs touristes que pour les utilisateurs locaux.

Le maintien voire le développement d'une activité écononigue en zone périphérique et dans le piénowt pyrénécn

Les flux touristiques engendrent une activité économique essentiellement liée à l'hébergement, la restauration, l'artisanat et aux services d'animation et de logistique des loisirs. Que ce soit dans les stations touristiques ou en périphérie de ces dernières, on assiste depuis les années 1980 à un développement, en plus desactivités traditionnelles d'hôtellerie et de restauration, d'activités très spécialisées, souvent caractérisées par une forte valeur ajoutée. Les plus notables sont les suivantes:

- activités liées au tourisme sportif et d'aventure;

- activités liées au tourisme de santé et de remise en forme (en liaison avec le thermalisme);

- activités liées au culturel;

- activités de transports spécialisés;

- activités d'information;

- activités liées à la déoouverte de pratiques artisanales;

Valorisation toteristigue de la zone péripberique et du Pienont par le Parc National des

Deux composantes de valorisation du système environnemental lié au Parc National des Pyrénées peuvent être dégagées de ce qui précède. Ces composantes ne prennent leur valeur que dans le maintien d'un niveau de tourisme équilibré et régulé.

Zone périphérique et Piémont pyténéen peuvent trouver dans les flux touristiques liés au Parc une valorisation de leurs domaines. L'identification de ces deux composantes estrelativement aiséecompte tenu de nos approches précédentes: systèmes.

Valorisation des hommes par acquisition de compétences et de métiers axés sur l'environnement naturel, valorisation du patrimoine immobilier existant, valorisation du patrimoine culturel, sont les caractéristiques de ce nouvel équilibre touristique. La diffusion des flux vers les zones périphériques et le piémont, nourrie par l'intérêt touristique majeur que représente leParc, assurel'essentiel de ce rééquilibrage de la fréquentation touristique et par là d'une activité économique à un niveau suffisant, compatibleaveclemaintien d'une
- activités de découverte des écomême, constitue la garantie de maintien 
vie dans ces zones. Cette activité à forte valeur ajoutéecaractérisée par un minimum de Déséconomies, devient un avantage économique et social décisif lorsqu'elle complète et soutient une activité traditionnelle locale liée au rythme de la nature (élevage, polyculture, exploitation forestière). Par son apport en trésorerie et en rentabilité, la démarche de multi-activité est un facteur clef de développement des vallées; il conviendrait toutefois que les cadres juridiques et fiscaux soient adaptés à cette nouvelle réalité et à la nouvelle dynamique engagée. Nous conclurons par une considération encourageante bien que non encore inscrite dans la durée: l'apparition debalances démographiques positivesdans les trois zones où elles ne l'étaient plus depuisdenombreusesannées:valléed'Aure, de Louron, de Barèges et d'Aspe.

Des compasants dimages fortes pour promouvoir une région

L'identificateur Parc National des Pyrénées est devenu une symbolique forte, utilisable par les collectivités du système environnemental lié au Parc. Cet identificateur peut se trouver renforcé par deux éléments complémentaires:

- un lien d'image avec le Parc National Espagnol d'Ordesa etMonte Perdido;

- la potentialité de pouvoir inscrire cet ensemble dans le cadre des actions de I'UNESCO, à savoir sa reconnaissance comme élément du patrimoine de l'humanité( ${ }^{(9)}$.

Les composantes d'images générées par l'idéede ParcNational ${ }^{(10)}$ des Pyrénées sont au nombre de trois dont deux interferent avec l'ensemble du massif pyrénéen:

- l'idée d'un espace préservé riche en flore et en faune;

- l'idée d'une zone frontière riche en potentialités de dépaysement;

- l'idée d'accessibilité, l'idée de montagnes «aimables».

Ces composantes sont souvent reprises et utilisées par bon nombre d'entités locales et régionales. Une étude documentaire, même superficielle, traduit l'impact réel que les praticiens de la communication attribuent à la mise en avant d'une telle référence. Citons quelques exemples:

- ville de Tarbes: brochure 1993 de présentation de la ville; 4 pages sur 43 traitent du Parc National des Pyrénées;

- ville de Pau: brochure 1992; 2 pages présentent le Parc;

- Fédération Thermale et Climatique: 2 paragraphes présentent le Parc;

- Organisation proposant des randonnées sur l'ensemble de la chaîne pyrénéenne ayant un siège de commercialisation à Paris: la référence au Parc National apparaît à 10 reprises dans les 80 pages que comporte son catalogue.

Ces quelques exemples relevés parmi beaucoup d'autres soulignent, si besoin était, tout l'impact attribué à l'image du Parc National des Pyrénées.

Ainsi, de nombreux acteurs du système environnemental lié au Parc utilisent très largement toute ou partie des composants d'images qu'il génère à un point tel que d'autres zones des Pyrénées non couvertes par un parc (Ariège, Cerdagne) envisagent de demander la mise en place de pares, un des arguments évoquéle plus souvent étant le «plus» d'image induit par l'existence d'un pare naturel.

\section{Conclusion}

À l'issue de ces quelques développements, il ressort que la dynamique de valorisation du système environnemental du Parc National des Pyrénées par un tourisme équilibré repose sur trois éléments:

- une typologie d'usagers du Parc et un effort pédagogique des institutions locales qui ont facilitél'appropriation par lesutilisateursdu Parcdesmesures de régulation de la fréquentation du Parc. Cela explique aussi la relative facilité avec laquelle les efforts de rééquilibrage de la fréquentation touristique sont mis en place et acceptés;

- un niveau d'activité suffisant, fondé sur la pluriactivité et tourné vers le Parc et les activités associées. Certes, des efforts restent à mener, particulièrement en zone périphérique, où il convient d'atteindre un niveau d'activité suffisant et compatible avec les volontés, toujours affirmées par les résidents, de vivre et travailler sur place:

- un retour d'images très positif qui favorise une large utilisation de l'étiquette Parc National des Pyrénées.
Les fréquentes références relevées dans les outils de communication confirment l'excellente tenųe des composantes d'imagedu Parc. À ceux qui en ont la charge de la compléter et de l'améliorer... $f$

\section{RÉFÉRENCES}

(1) M. Bourget, F. Victor, J. Tomatis, J.J. Maiftait et P. Peron, La frequentation touristique du Parc National des Pyrénbes, Documents scientifiques du Parc National des Pyrénées.

(2) Nous pensons pour l'essentiel aux tensions engendrées par les projets et déweloppements routiers en vallée d'Aspe.

(3) Ces trois activités allaient soument de pair.

4) Certains spécialistes affirment que les années 1950 ont éte cruciales pour la survie de l'ours. Soixante dix ours recensés en 1954, probablement plusieurs centaines au début du siecle (article Jean Ricaud - Numéro Spécial de GEO sur les Pyrénées 1987). Certes le développement de la fréquentation touristique etl'ouverture des routes dans le massif (pastorales ou d'altitude) a pu accelérer marginalement sa disparition mais le seuli critique de non maintiende la population était atteint bien avant le développement touristique des Pyrénées.

15) Le terme nature est bien tuidement pris ici dans ses dewx acceptions.

(6) M. Bourget, F. Victor, J. Tomatis, J.J. Malfait et $P$. Peron, Op. eit., Documents Scientifiques du Parc National des Pyrénées.

(7) Etude de Monique Morales Irsam, Université des Pays de l'Adour, 1992.

(8) M. Bourget, F, Victor, J. Tomatis, J.J. Malfait et P. Peron, Op. cit.. Documents Scientifiques du Parc National.

(9) Voir àce sujet l'action mente par le Guide de Haute Montagne, Patrice Debelleton, auteur de nombreux ouvrages sur les Pyrénées et défenseur du projet.

(10) Etude réalisée par des étudiants de maitrise Hotellerie, Tourisme, Thermalisme - CETIA 1993. 